Research Article

Animal Genetics

\title{
Comparative analyses of three swallow species (Aves, Passeriformes, Hirundinidae): Insights on karyotype evolution and genomic organization
}

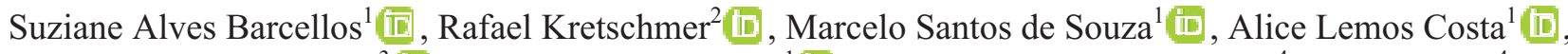
Tiago Marafiga Degrandi ${ }^{3}$, Cassiane Furlan Lopes ${ }^{1}$ (i], Malcolm A. Ferguson-Smith ${ }^{4}$, Jorge Pereira ${ }^{4}$, Edivaldo Herculano Correa de Oliveira ${ }^{5,6}$ [i], Ricardo José Gunski ${ }^{1}$ [D, Analía del Valle Garnero ${ }^{1}$ [

${ }^{1}$ Universidade Federal do Pampa, Programa de Pós-graduação em Ciências Biológicas - PPGCB, São Gabriel, RS, Brazil.

${ }^{2}$ Universidade Federal do Rio Grande do Sul, Programa de Pós-graduação em Genética e Biologia Molecular - PPGBM, Porto Alegre, RS, Brazil.

${ }^{3}$ Universidade Federal do Paraná, Programa de Pós-Graduação em Genética, PPGG, Curitiba, PR, Brazil.

${ }^{4}$ University of Cambridge Department of Veterinary Medicine, Cambridge Resource Centre for Comparative Genomics, Cambridge, United Kingdom.

${ }^{5}$ Universidade Federal do Pará, Instituto de Ciências Exatas e Naturais, Belém, PA, Brazil.

${ }^{6}$ Instituto Evandro Chagas, Laboratório de Cultura de Tecidos e Citogenética, Ananindeua, PA, Brazil.

\begin{abstract}
Despite the richness of species in the Hirudinidae family, little is known about the genome organization of swallows. The Progne tapera species presents genetic and morphological difference when compared to other members of the same genus. Hence, the aims of this study were to analyze the chromosomal evolution of three species Progne tapera, Progne chalybea and Pygochelidon cyanoleuca - by comparative chromosome painting using two sets of probes, Gallus gallus and Zenaida auriculata, in order to determine chromosome homologies and the relationship between these species. All karyotypes exhibited 76 chromosomes with similar morphology, except for the 5th, 6th and 7th chromosome pairs in P. cyanoleuca. Additionally, comparative chromosome painting demonstrated the same hybridization pattern in the two Progne, which was similar to the putative avian ancestral karyotype, except for the centric fission in the first pair, as found in other Passeriformes. Thus, these data display a close relationship between the Progne species. Although $P$. cyanoleuca demonstrated the same fission in the first pair of the ancestral syntenic (GGA1), it also showed an additional chromosomal rearrangement for this species, namely a fusion with a microchromosome in the seventh pair.
\end{abstract}

Keywords: Homology, molecular cytogenetics, fluorescent in situ hybridization, Hirundinidae.

Received: July 15, 2019; Accepted: December 19, 2019.

\section{Introduction}

The order Passeriformes is one the most diverse within the class Aves, including around 6000 species (Del Hoyo et al., 2011). As the other members from this class, it presents small genome, high chromosomal number, a few pairs of macrochromosomes and several microchromosome pairs. Furthermore, birds have a sexual system ZZ/ZW, where the female is heterogametic (Griffin et al., 2007; Barcellos et al., 2019).

The Hirundinidae family (Aves: Passeriformes), commonly known as swallows, comprises approximately 84 species (Dickinson, 2003; Sheldon et al., 2005). These birds are well known worldwide due to their cosmopolitan

Send correspondence to Ricardo J. Gunski. Universidade Federal do Pampa, Programa de Pós-graduação em Ciências Biológicas PPGCB, Av. Antonio Trilha, 1847, São Gabriel, 97300-162, RS, Brazil. E-mail: gunski@yahoo.com.br. habits, behavior and ecology (Sheldon et al., 2005). Moreover, they are migratory and insectivorous. Due to the scarcity of food resources in winter, swallows tend to fly several miles to find food and a safe place to stay during this season (Sigrist, 2013).

Progne tapera (Linnaeus, 1766), Progne chalybea (Gmelin, 1789) and Pygochelidon cyanoleuca (Vieillot, 1817) have similar karyotypical organization with the same diploid number $(2 n=76)$ and distribution of repetitive DNA. Furthermore, recent studies with these species found an interesting characteristic, an enlarged $\mathrm{W}$ chromosome (Barcellos et al., 2019). Despite recent research, the cytogenetics of swallows is still poorly defined.

Cross-species chromosome painting has been applied widely for evolutionary biology studies and karyotype evolution (Ferguson-Smith and Trifonov, 2007; Ellegren, 2010) and, in particular, to identify chromosomal homo- 
logies in passerines species (Kretschmer et al., 2018a). The most common probes used for analyses in birds are from Gallus gallus (Linnaeus, 1758) (GGA) and Leucopternis albicollis (Latham, 1790) (LAL) (Griffin et al., 2007; de Oliveira et al., 2010). Using GGA and LAL probes in Passeriformes revealed a fission and numerous inversions in the first chromosome pair of the ancestral syntenic (GGA1), an apomorphy that has been seen in all species belonging to this order analyzed by fluorescent in situ hybridization (FISH) (Kretschmer et al., 2014, 2015, 2018a; dos Santos et al., 2015, 2017).

Recently, a new set of whole chromosome-specific probes was developed using Zenaida auriculata species (Des Murs, 1847). This probe set has the similar organization pattern of macrochromosomes as the putative ancestral karyotype of birds (PAK) and is also similar to that of $G$. gallus. Furthermore, it shows interchromosomal rearrangements, which are extremely important for karyotype evolution (Kretschmer et al., 2018b).

In order to study the genome organization of Hirundinidae, and the relationship between them, we present here for the first time chromosome painting using whole-chromosome probes of G. gallus and Z. auriculata in three species of swallows, $P$. chalybea, $P$. tapera and $P$. cyanoleuca.

\section{Material and Methods}

\section{Species}

The present work examines twelve individuals belonging to the Hirundinidae family: $P$. chalybea ( 3 females and 2 males); $P$. tapera ( 2 females and 2 males) and $P$. cyanoleuca (3 females), all collected in São Gabriel - Rio Grande do Sul State, Brazil (SISBIO Permission Number: 33860-4). The protocols were approved by the Ethics Committee on the use of animals (CEUA- Universidade Federal do Pampa, 026/2012).

\section{Chromosome isolation}

Chromosomes were obtained by fibroblast culture (Sasaki et al., 1968) and short-term bone marrow culture (Garnero and Gunski, 2000). The procedures included: hypotonic treatment, incubation with colchicine $(0,05 \%)$ and cell fixation using methanol and acetic acid (3:1). Diploid number and chromosome morphology were determined from the analyses of 30 mitotic cells stained with Giemsa 5\% in $0.07 \mathrm{M}$ phosphate buffer, $\mathrm{pH}$ 6.8. Subsequently, metaphases were analyzed by microscopy.

\section{Fluorescent in situ hybridization (FISH)}

Chromosome painting utilized two sets of probes: $Z$. auriculata (ZAU 1-8 and Z) and G. gallus (GGA 9-10). Comparisons were based on homology between ZAU and GGA (Kretschmer et al., 2018b). Protocols for hybridization were performed as described in de Oliveira et al. (2010). The FISH results were examined by epifluorescent microscopy.

\section{Results}

\section{Karyotype analyses}

The diploid number is 76 for the three swallow species, which corroborate previous studies (Barcellos et al., 2019). The $1 \mathrm{st}, 4$ th, 10 th pairs and also the $\mathrm{Z}$ chromosome are metacentric, pairs 2 and 3 are acrocentric, while 8th, 9th, 11th and all microchromosomes are telocentric. Only three morphological differences between species were observed: in P. cyanoleuca, the 5th chromosome pair is acrocentric, the 6th submetacentric, and the 7th metacentric, whereas in the Progne species the 5th pair is submetacentric and the 6th and 7th chromosome pairs are telocentric. The $\mathrm{W}$ sex chromosome is submetacentric in all the three species (Figure 1).

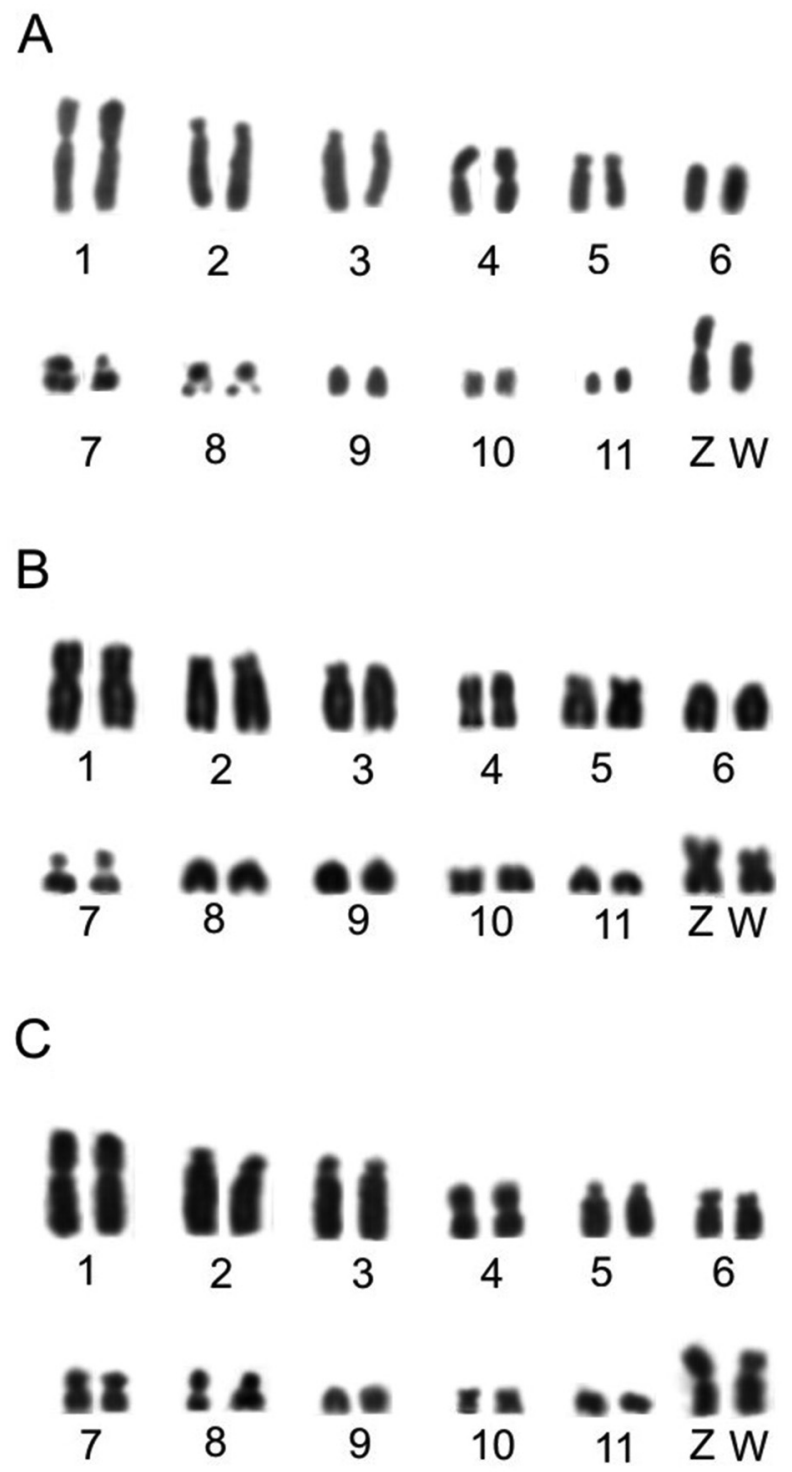

Figure 1 - Partial karyotype of three females with conventional staining (Giemsa). $\mathrm{A}=P$. tapera, $\mathrm{B}=P$. chalybea and $\mathrm{C}=P$. cyanoleuca . 


\section{Chromosome painting using GGA and ZAU probes}

GGA and ZAU probes revealed conservation of some syntenic groups in swallows. ZAU1 hybridized in the second and fifth chromosome pairs, while ZAU2 demonstrated hybridization signals only in the first pair in the three swallow species (Figure $2 \mathrm{~A}, \mathrm{D}$ and G). The hybridization patterns using GGA and ZAU probes were the same for $P$. tapera and P. chalybea. Hybridization experiments demonstrated that GGA1 (ancestral chromosome 1) corresponds to two distinct chromosome pairs and GGA2, 3, 4, $5,6,7,8,9$ and 10 each correspond to one pair in swallows (Figure $3 \mathrm{~A}$ and B). P. cyanoleuca exhibited a similar pattern of hybridization, except for the seventh pair of chromo-

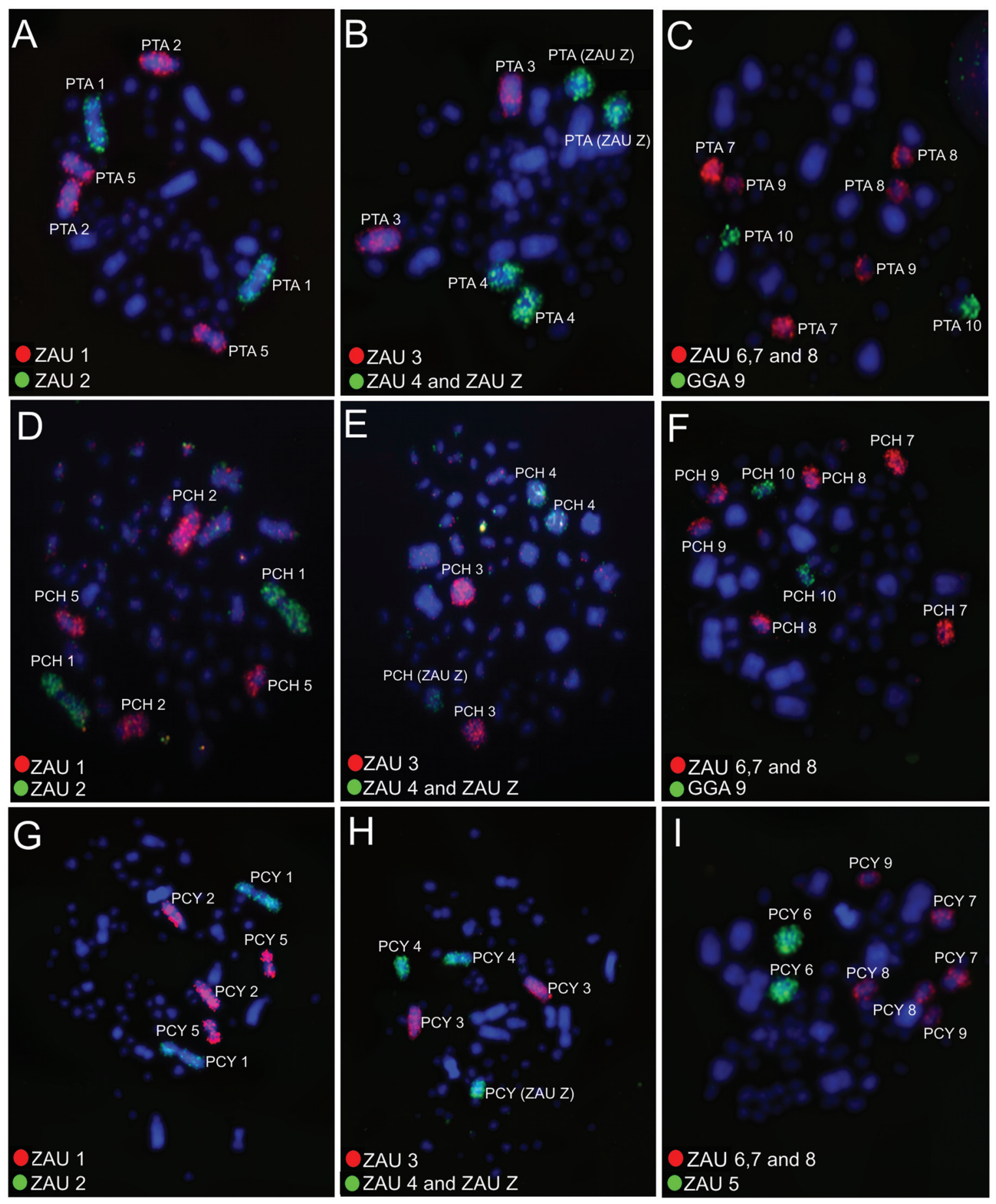

Figure 2 - FISH experiments using Gallus gallus (GGA) and Zenaida auriculata (ZAU) probes hybridized onto P. tapera - PTA (A-C), P. chalybea PCH (D-F) and P. cyanoleuca - PCY (G-I) metaphases. Probes are indicated in the lower left corner of each image; probes were labeled with biotin/Cy3 (red) or fluorescence (green). 


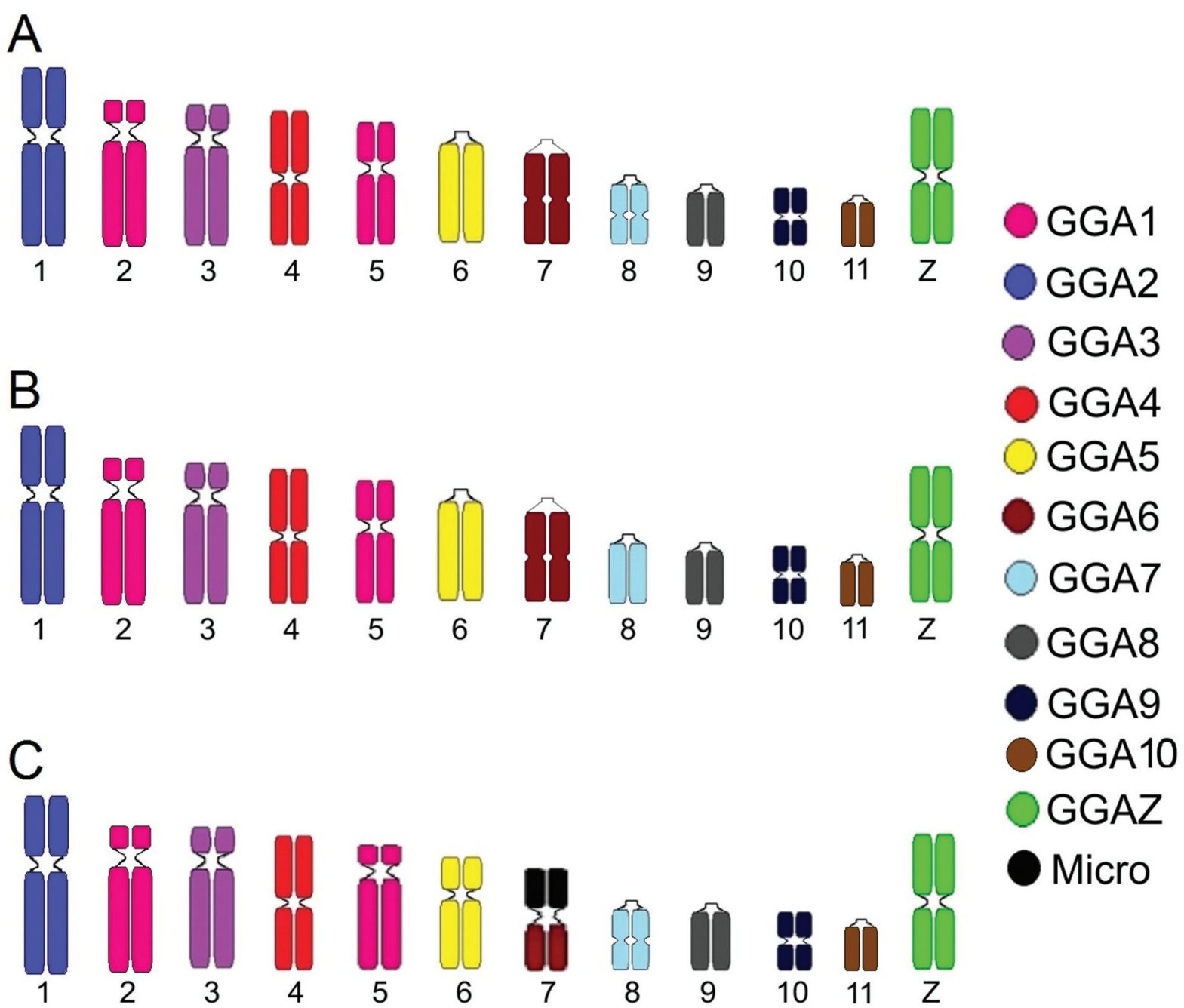

Figure 3 - Homology map between G. gallus and three swallow species: P. tapera (A) and P.chalybea (B), P. cyanoleuca (C).

somes, which shows fusion with a microchromosome (Figures 2 I and 3 B).

\section{Discussion}

The swallow karyotypes presented a diploid number and morphology typically found in most Passeriformes (Tegelström and Ryttman, 1981; Santos and Gunski, 2006), with 76 chromosomes and many microchromosome pairs, as observed in Barcellos et al. (2019). However, these species have a $\mathrm{W}$ chromosome relatively bigger than the $\mathrm{W}$ of most Passeriformes species due to a highly repetitive sequences accumulation, such as microsatellites (Berlin and Ellegren, 2004; Chen et al., 2012; Zhang et al., 2014; Barcellos et al., 2019).

The Neognathae group, so called modern aves, is divided in two distinct clades: Galloanseres and the Neoaves. G. gallus belongs to Galloanseres, considered a basal group and very similar to PAK. On the other hand, Z. auriculata is a Neoaves member, so it is closer to derived groups (Jarvis et al., 2014). Despite the phylogenetic distance, there is a high similarity between GGA and ZAU. Since studies using both probes showed the same chromosomal homologies in both, except for GGA4, which has a fusion between two ancestor chromosomal pairs (PAK4+10), while ZAU shows fission (ZAU4-11) (Kretschmer et al., 2018 b). However, the use of ZAU probes showed clear hybridization signals in derived species (Kretschmer et al., 2020), which can also be observed in the swallows, probably due to their phylogenetic proximity.

The main differences found when compared to the putative ancestral avian karyotype (Griffin et al., 2007), is the fission in the first chromosome pair into two distinct pairs of macrochromosomes ( 2 and 5 pairs) and the fusion of a microchromosome in the seventh pair in $P$. cyanoleuca. The fission of the GGA 1 chromosome has been observed in all Passeriformes studied by FISH so far (Guttenbach et al., 2003; Derjusheva et al., 2004; Kretschmer et al., 2014, 2015, 2018a; dos Santos et al., 2015). In addition, this rearrangement can also be seen in most Accipitriformes, as well as in Psittaciformes 
(Kretschmer et al., 2018a), suggested as Passeriformes sister group (Hackett et al., 2008; Jarvis et al., 2014).

Among the swallows analyzed by FISH, only one chromosomal rearrangement was observed, a fusion with a microchromosome pair in $P$. cyanoleuca, which is the most derived species. This fusion had possibly occurred due to evolutionary pressures leading to interchromosomal and intrachromosomal rearrangements (Ellegren, 2010). Fusions among macrochromosomes and microchromosomes were also observed in two species of the genus Falco (Accipitriformes) (Kretschmer et al., 2018a). Although highly variable among lineages, intrachromosomal rearrangements might be significant contributors to the genetic and phenotypic diversity presented by the members of the class Aves (Damas et al., 2019).

The chromosome painting patterns found in swallows are very distinct when compared to distant bird orders, such as Galliformes and Anseriformes, which in general present chromosomal structure similar to PAK1-10 (Guttenbach et al., 2003; Shibusawa et al., 2003; Kretschmer et al., 2018a). The same occurs when compared with Gruiformes, Eurypygiformes and Trogoniformes, which show fusions in distinct macrochromosomes pairs and also some peculiar chromosomal fission for each order (Nanda et al., 2011; Furo et al., 2015; Degrandi et al., 2017).

In the past, the Hirundinidae family had some phylogenetic issues in relation to $P$. tapera due to the phenotypic and genetic difference from other Progne species (Moyle $e t$ al., 2008). Nevertheless, recent studies using classical and molecular cytogenetics have shown a greater similarity between the $P$. tapera and $P$. chalybea when compared to $P$. cyanoleuca (Barcellos et al., 2019). Taken together, these examples and our data support the current phylogeny of the genus Progne, which puts all Progne species into a single genus (Sheldon et al., 2005; Moyle et al., 2008; Barcellos et al., 2019).

Overall, the analyses allow us to identify homologies between PAK and three swallow species using GGA and ZAU probes, providing data about the mechanisms involved in karyotype evolution in the Hirundinidae family. Moreover, FISH experiments played an important role in identifying chromosomal rearrangements, such as the microchromossome and macrochromosome fusion in $P$. cyanoleuca species, which clarifies the relationships among the swallows.

\section{Acknowledgments}

We would like to thank all colleagues from the Grupo de Pesquisa Diversidade Genética Animal (Universidade Federal do Pampa) and Cambridge Resource Centre for Comparative Genomics Centre for Veterinary Science (University of Cambridge) for technical and institutional support. This study was financed in part by the Coordenação de Aperfeiçoamento de Pessoal de Nível Superior - Brasil (CAPES) - Finance Code 001.

\section{Conflict of Interest}

The authors declare that there is no conflict of interest that could be perceived as prejudicial to the impartiality of the reported research.

\section{Author Contributions}

SAB, RK, MSS, ALC, RJG and AVG conceived and designed the study; SAB, RK, MSS, ALC, MAF and JP conducted the experiments; SAB, RK, MSS, ALC, MAF, JP, RJG and AVG analyzed the data; SAB, RK, MSS, ALC, TMD, CFL, MAF, EHCO, RJG and AVG wrote, reviewed and edited the manuscript; AVG and RJG supervised the study, and all authors read and approved the final version.

\section{References}

Barcellos SA, Kretschmer R, de Souza MS, Costa AL, Marafiga TD, dos Santos M, de Oliveira EHC, Cioff MB, Gunski RJ and Garnero ADV (2019) Karyotype evolution and distinct evolutionary history of the $\mathrm{W}$ chromosomes in swallows (Aves, Passeriformes). Cytogenet Genome Res 158:98-105.

Berlin S and Ellegren H (2004) Chicken W: a genetically uniform chromosome in a highly variable genome. Proc Natl Acad Sci U S A 101:15967-15969.

Chen N, Bellott DW, Page DC and Clark AG (2012) Identification of avian W-linked contigs by short-read sequencing. BMC Genomics 13:183.

Damas J, O'Connor RE, Griffin DK and Larkin DM (2019) Avian chromosomal evolution. In: Kraus RHS (eds) Avian Genomics in Ecology and Evolution. Springer, Cham, pp 69-92.

de Oliveira EHC, Tagliarini MM, Rissino JD, Pieczarka JC, Nagamachi CY, O'Brien PCM and Ferguson-Smith MA (2010) Reciprocal chromosome painting between white hawk (Leucopternis albicollis) and chicken reveals extensive fusions and fissions during karyotype evolution of Accipitridae (Aves, Falconiformes). Chromosome Res 18:349-355.

Degrandi TM, Garnero ADV, O'Brien PCM, Ferguson-Smith MA, Kretschmer R, de Oliveira EHC and Gunski RJ (2015) Chromosome painting in Trogon s. surrucura (Aves, Trogoniformes) reveals a karyotype derived by chromosomal fissions, fusions, and inversions. Cytogenet Genome Res 151:208-215.

Del Hoyo J, Elliott A and Christie AD (2011) Handbook of the Birds of the World. 16th edition. Lynx Edicions, Barcelona, 894 pp.

Derjusheva S, Kurganova A, Haberman F and Gaginskaia E (2004) High chromosome conservation detected by comparative chromosome painting in chicken, pigeon and passerine birds. Chromosome Res 12:715-723.

Dickinson EC (2003) The Howard \& Moore complete checklist of the birds of the world. 3rd edition. Princeton University Press, New Jersey, 622 pp.

dos Santos MDS, Kretschmer R, Frankl-Vilches C, Bakker A, Gahr M, Ferguson-Smith MA and de Oliveira EH (2017) Comparative cytogenetics between two important songbirds: The zebra finch and the canary. PloS One 12:e0170997. 
dos Santos MS, Kretschmer R, Silva FA, Ledesma MA, O'Brien PCM, Ferguson-Smith MA, Garnero ADV, de Oliveira EH and Gunski RJ (2015) Intrachromosomal rearrangements in two representatives of the genus Saltator (Thraupidae, Passeriformes) and the occurrence of heteromorphic $\mathrm{Z}$ chromosomes. Genetica 143:535-543.

Ellegren H (2010) Evolutionary stasis: the stable chromosomes of birds. Trends Ecol Evol 25:283-291.

Ferguson-Smith MA and Trifonov V (2007) Mammalian karyotype evolution. Nat Rev Genet 8:950-962.

Furo IO, Monte AA, dos Santos MS, Tagliarini MM, O'Brien PCM, Ferguson-Smith MA and de Oliveira EH (2015) Cytotaxonomy of Eurypyga helias (Gruiformes, Eurypygidae): First karyotypic description and phylogenetic proximity with Rynochetidae. PLoS One 10:e0143982.

Garnero AV and Gunski RJ (2000) Comparative analysis of the karyotype of Nothura maculosa and Rynchotus rufescens (Aves: Tinamidae) A case of chromosomal polymorphism. Nucleus 43:64-70.

Griffin DK, Robertson LBW, Tempest HG and Skinner BM (2007) The evolution of the avian genome as revealed by comparative molecular cytogenetic. Cytogenet Genome Res 117:64-77.

Guttenbach M, Nanda I, Feichtinger W, Masabanda JS, Griffin DK and Schmida M (2003) Comparative chromosome painting of chicken autosomal paints 1-9 in nine different bird species. Cytogenet Genome Res 103:173-184.

Hackett SJ, Kimball RT, Reddy S, Bowie RCK, Braun EL, Braun MJ, Chojnowski JL, Cox WA, Han KL, Harshman J et al. (2008) A phylogenomic study of birds reveals their evolutionary history. Science 320:1763-1768.

Jarvis ED, Mirarab S, Aberer AJ, Li B, Houde P, Li C, Ho SY, Faircloth BC, Nabholz B, Howard JT et al. (2014) Wholegenome analyses resolve early branches in the tree of life of modern birds. Science 346:1320-1331.

Kretschmer R, Gunski RJ, Garnero ADV, Furo IO, O'Brien PCM, Ferguson-Smith MA and de Oliveira EHC (2014) Molecular cytogenetic characterization of multiple intrachromosomal rearrangements in two representatives of the genus Turdus (Turdidae, Passeriformes). PLoS One 9:e103338.

Kretschmer R, de Oliveira EHC, dos Santos MS, Furo IO, O'Brien PCM, Ferguson-Smith MA, Garnero ADV and Gunski RJ (2015) Chromosome mapping of the large Elaenia (Elaenia spectabilis): evidence for a cytogenetic signature for passeriform birds? Biol J Linn Soc 115:391-398.
Kretschmer R, Ferguson-Smith MA and de Oliveira EHC (2018a) Karyotype evolution in birds: From conventional staining to chromosome painting. Genes 9:181.

Kretschmer R, de Oliveira Furo I, Gunski RJ, Garnero ADV, Pereira JC, O'Brien PCM, Ferguson-Smith MA, de Oliveira EHC and de Freitas TRO (2018b) Comparative chromosome painting in Columbidae (Columbiformes) reinforces divergence in Passerea and Columbea. Chromosome Res 26:211-223

Moyle RG, Slikas B, Whittingham LA, Winkler DW and Sheldon FH (2008) DNA sequence assessment of phylogenetic relationships among New World martins (Hirundinidae: Progne). Wilson J Ornithol 120:683-691.

Nanda I, Benisch P, Fetting D, Haaf T and Schmid M (2011) Synteny conservation of chicken macrochromosomes 1-10 in different Avian lineages revealed by cross-species chromosome painting. Cytogenet Genome Res 132:165-181.

Santos LP and Gunski RJ (2006) Revisão de dados citogenéticos sobre a avifauna brasileira. Rev Bras Ornitol 14:35-45.

Sasaki M, Ikeuchi T and Makino S (1968) A feather pulp culture technique for avian chromosomes, with notes on the chromosomes of the peafowl and the ostrich. Experientia 24:1292-1293.

Sheldon FH, Whittingham LA, Moyle RG, Slikas B and Winkler DW (2005) Phylogeny of swallows (Aves: Hirundinidae) estimated from nuclear and mitochondrial DNA sequences. Mol Phylogenet Evol 35:254-270.

Shibusawa M, Nishibori M, Nishida-Umehara C, Tsudzuk M, Masaband J, Griffin DK and Matsuda Y (2004) Karyotypic evolution in the Galliformes: An examination of the process of karyotypic evolution by comparison of the molecular cytogenetic findings with the molecular phylogeny. Cytogenet Genome Res 106:111-119.

Sigrist T (2013) Guia de campo Avis Brasilis: Avifauna Brasileira. 3rd edition. Avis Brasilis Editora, São Paulo, 608 pp.

Tegelström H and Ryttman H (1981) Chromosomes in birds (Aves): evolutionary implications of macro- and microchromosome numbers and lengths. Hereditas 94:225-233.

Zhang G, Li C, Li Q, Li B, Larkin DM, Lee C, Storz JF, Antunes A, Greenwold MJ, Meredith RW et al. (2014) Comparative genomics reveals insights into avian genome evolution and adaptation. Science 346:1311-1320.

Associate Editor: Louis Klaczko

License information: This is an open-access article distributed under the terms of the Creative Commons Attribution License (type CC-BY), which permits unrestricted use, distribution and reproduction in any medium, provided the original article is properly cited. 